\title{
VARIATIONAL PRINCIPLES, DUALITY, LEGENDRE TRANSFORMATIONS AND MINE SHAFT VENTILATION
}

\author{
A. M. WATTS ${ }^{1}$ \\ (Received 11 September 1991; revised 17 January 1992)

\begin{abstract}
The calculation of flows in pipe networks and in networks of mine shafts and the calculations of the currents in electrical circuits can be represented as variational problems. There are two approaches: the nodal method and the loop method. There is a variational representation for each of these. This paper describes the relationship between the two representations and in particular shows that the loop formulation is the Wolfe dual of the nodal formulation after the application of Legendre transformations to the variables and to the objective function.
\end{abstract}

\section{Introduction}

Flow in pipe networks, mine shaft ventilation and some aspects of electrical circuits are analogous and their theoretical treatments are very similar. In this paper we think in terms of mine shaft ventilation.

As described by Osiadacz [3], there are two alternative methods which can be used to solve pipe-flow equations: the nodal method and the loop method. The loop method seems to be preferred by practitioners because it is slightly more efficient. It does not seem to have been pointed out in the literature that both the nodal and loop methods, at least in many applications, can be expressed in terms of variational principles and that one is the dual of the other. This paper describes the variational principles and the relationships between them. One of the interesting aspects of the transformation from the primal to the form of the dual problem that is used, is that Legendre

\footnotetext{
${ }^{1}$ Centre for Industrial and Applied Mathematics and Parallel Computing, Mathematics Department, The University of Queensland, Qld 4072.

(C) Copyright Australian Mathematical Society 1992, Serial-fee code 0334-2700/92
} 
transformations are used for both the variables and the objective function. Most readers will be familiar with Legendre transformations in two contexts: the thermodynamics and canonical transformations.

Traditionally, the loop method has been used for calculation of mine shaft ventilation. The solution of the pressure-balance equations has been done using a relaxation method known as the Hardy-Cross method. More recently, Wang [4] has used a algorithm based on the Newton method.

One question which arises is whether the loop method used for pipe flow and mine shaft ventilation should be used for power-flow calculations in large electrical power networks. These are typically solved using a method analogous to the nodal method, although with direct current circuits the loop method is apparently frequently used. In a power network there are reactive components, i.e. inductances and capacitors, so that the structure of the problems could be significantly different. For example, it is not clear in those cases whether the variational principle would be strictly a minimisation and whether the objective function would be convex. This is a topic for further investigation.

\section{Statement of the problem}

For the purposes of this work, the network of mine shafts is considered to be closed except for an input node and an output node where the pressure is specified. In reality, one of these might be open to the atmosphere and at the other there might be a pump which maintains a constant pressure. The theory could be extended to various other connections to the outside world or to various applications of pumps inside the network.

An important condition for this theory is that the flow in an arc be dependent only on the pressure difference from one end to the other.

The network has $n$ nodes and arcs connecting them. There is not necessarily an arc between each pair of nodes, but the network must be connected. The arcs are directed (but can have negative flows). The "from" node for arc number $k$ is denoted by $\mathscr{F}_{k}$ and the "to" node by $\mathscr{T}_{k}$. The flow on arc $k$ is $q_{k}$ and the pressure difference between the from and to nodes is $p_{k}$, i.e. $p_{k}=P_{i}-P_{j}$, where $i=\mathscr{F}_{k}, j=\mathscr{F}_{k}$ and $P_{i}$ is the pressure at node $i$.

The net flow into each node is zero except for the input node number 1 and the output node number $n$. At these nodes, the pressures are specified, or at least their difference is specified by

$$
P_{1}-P_{n}=P_{0}
$$


The pressure and flow are assumed to be related by the dynamic equations

$$
q_{k}=f_{k}\left(p_{k}\right), \quad \text { where } f_{k}^{\prime}>0 \text {. }
$$

A typical relationship would be

$$
p_{k}=\alpha_{k}\left|q_{k}\right| q_{k}+\beta_{k} q_{k}
$$

This form covers many of the applications but there are some, such as flow of gases at high speeds, or with large pressure changes where compressibility effects are important, or the flow of fluids such as steam, where changes of phase can occur. In those cases, something more general would be required for a realistic representation, and it is not clear at this stage how the theory would extend to them.

The aim then is to find $p_{k}$ and $q_{k}$ which satisfy the dynamic equations, subject also to pressure consistency (Kirchoff's law), to the specified input and output pressures and to the conditions of zero net flow into each of the internal nodes:

$$
\sum c_{k} p_{k}=0
$$

where the sum is taken over all arcs in any cycle in the network and $c_{k}= \pm 1$ depending on whether arc $k$ is in the direction of the cycle or the opposite direction,

$$
\sum c_{k} p_{k}=P_{1}-P_{n}
$$

where the sum is over all arcs in any path from the input node to the output node, and for each node $i$,

$$
\sum_{k \mid \mathscr{F}_{k}=i} q_{k}-\sum_{k \mid \mathscr{F}_{k}=i} q_{k}=0
$$

Here, the first summation is over all arcs whose from node is $i$ and in the second over all arcs whose to node is $i$.

\section{Nodal method}

We define a new function $\phi_{k}$ by

$$
\phi_{k}\left(p_{k}\right)=\int_{0}^{p_{k}} f_{k}(u) d u,
$$

so that $\phi_{k}^{\prime}=q_{k}$ and $\phi_{k}^{\prime \prime}>0$. This is analogous to a potential in an electrical problem. If all the pressure differences $p_{k}$ are expressed in terms of the node pressures $P_{i}$, pressure consistency is automatically achieved. We then need to adjust the node pressures so that the node flow conditions are satisfied. 
This nodal formulation is then equivalent to the variational problem:

$$
\begin{aligned}
& \text { Minimise } F(\mathbf{P})=\sum_{k, i=\mathscr{F}_{k}, j=\mathscr{F}_{k}} \phi_{k}\left(P_{i}-P_{j}\right) \\
& \text { subject to } P_{1}-P_{n}=P_{0} .
\end{aligned}
$$

Since $\phi_{k}^{\prime \prime}$ is positive, $F$ is a convex function and there is a unique minimum to the variational problem. Because of the convexity, there is also a Wolfe dual. (See, for example, Fletcher [2].) Define the Lagrangian

$$
\begin{aligned}
\mathscr{L}(\mathbf{P}, \lambda) & =F(\mathbf{P})-\lambda\left(P_{1}-P_{n}-P_{0}\right) \\
& =\sum_{k, i=\mathscr{F}_{k}, j=\mathscr{G}_{k}} \phi_{k}\left(P_{i}-P_{j}\right)-\lambda\left(P_{1}-P_{n}-P_{0}\right) .
\end{aligned}
$$

The dual is then:

$$
\begin{aligned}
& \text { Minimise } \mathscr{L}(\mathbf{P}, \lambda) \\
& \text { subject to } \nabla_{\mathbf{P}} \mathscr{L}=0 .
\end{aligned}
$$

Note that since the constraint in the primal problem is an equality one, $\lambda$ is not constrained to be positive in the dual problem. The constraint (12) ensures that the node flow conditions are satisfied. To solve the dual problem, it is necessary to characterise all the node pressures which satisfy these conditions. Since that is a difficult thing to do, it is natural to try to transform the dual into one using arc flows instead of node pressures. When this is done, the method becomes equivalent to the traditional loop method as described in Osiadacz, except that here it is presented as a variational principle.

\section{Transformation to loop formulation}

Firstly, a Legendre transformation is used to derive a replacement function for $\phi_{k}$. Put

$$
q_{k}=\phi_{k}^{\prime}\left(p_{k}\right)
$$

and then

$$
\psi_{k}\left(q_{k}\right)=p_{k} \phi_{k}^{\prime}\left(p_{k}\right)-\phi_{k}=p_{k} q_{k}-\phi_{k}\left(p_{k}\right)
$$

We then have

$$
p_{k}=\psi_{k}^{\prime}\left(q_{k}\right) \text {. }
$$

Strictly speaking, we do not need to know that the $q_{k}$ are flow variables. They could be thought of simply as abstract quantities. 
A similar transformation can be used to form a new objective function. Firstly, as in (11), define

$$
r_{i}=\frac{\partial \mathscr{L}}{\partial P_{i}}= \begin{cases}\sum_{k \mid \mathscr{F}_{k}=i} \phi_{k}^{\prime}\left(p_{k}\right)-\sum_{k \mid \mathscr{F}_{k}=i} \phi_{k}^{\prime}\left(p_{k}\right) & \text { for } i \neq 1, n, \\ \sum_{k \mid \mathscr{F}_{k}=i} \phi_{k}^{\prime}\left(p_{k}\right)-\sum_{k \mid \mathscr{F}_{k}=i} \phi_{k}^{\prime}\left(p_{k}\right)+\lambda & \text { for } i=n, \\ \sum_{k \mid \mathscr{F}_{k}=i} \phi_{k}^{\prime}\left(p_{k}\right)-\sum_{k \mid \mathscr{F}_{k}=i} \phi_{k}^{\prime}\left(p_{k}\right)-\lambda & \text { for } i=1 .\end{cases}
$$

It is clear from these expressions for the $r_{i}$ that the Lagrange multiplier $\lambda$ is the external flow out of node $n$ and the external flow into node 1 . If we introduce a dummy arc from node $n$ to node 1 , the network is closed and $\lambda$ is replaced by the flow on that arc. This is done when formulating the loop method below.

Then, in analogy with (12), put

$$
\begin{aligned}
\mathscr{H} & =\sum_{i} P_{i} \frac{\partial \mathscr{L}}{\partial P_{i}}-\mathscr{L} \\
& =\sum_{i} P_{i} r_{i}-\sum_{k} \phi_{k}\left(p_{k}\right)+\lambda\left(P_{1}-P_{n}-P_{0}\right) .
\end{aligned}
$$

This transformation has the appearance of the definition of the Hamiltonian in terms of a Lagrangian in mechanics but it is not clear whether the analogy goes further. One clear difference is that Hamilton's principle in mechanics is not in general a minimisation; it only defines a stationary point.

From the expressions for $r_{i}$ above we get:

$$
\begin{aligned}
\sum_{i} P_{i} r_{i} & =\sum_{i} P_{i}\left\{\sum_{k \mid i=\mathscr{F}_{k}} \phi_{k}^{\prime}\left(p_{k}\right)-\sum_{k \mid u=\mathscr{T}_{k}} \phi_{k}^{\prime}\left(p_{k}\right)\right\}+\lambda P_{n}-\lambda P_{1} \\
& =\sum_{k, i=\mathscr{F}_{k}, j=\mathscr{G}_{k}}\left(P_{i}-P_{j}\right) \phi_{k}^{\prime}\left(P_{i}-P_{j}\right)-\lambda\left(P_{1}-P_{n}\right) \\
& =\sum_{k}\left\{\phi_{k}\left(p_{k}\right)+\psi_{k}\left(q_{k}\right)\right\}-\lambda\left(P_{1}-P_{n}\right) .
\end{aligned}
$$

Hence,

$$
\mathscr{H}=\sum_{k} \psi_{k}\left(q_{k}\right)-\lambda P_{0}
$$

At optimality, $r_{i}=0$ and $\mathscr{H}=-\mathscr{L}$. Therefore, if $\mathscr{L}$ takes a maximum value, $\mathscr{H}$ takes a minimum value. The variational procedure for the loop 
method is then to minimise $\mathscr{H}$ subject to $r_{i}=0$. This is also a convex objective function.

\section{The loop formulation}

It is still necessary to characterise the arc flows $q_{k}$ so that the constraints on the dual problem are satisfied, i.e. so that the net flow into each of the nodes is zero. An artificial arc is added first from the output node to the input node. Then all nodes must have zero net flow and the flow on the artificial arc is $\lambda$. The standard method (see Osiadacz) is to consider a complete set of cycles in the extended network and use the flows on the cycles as variables. The arc flow is then the superposition of the cycle flows for cycles which contain the arc.

One way of constructing a complete set of cycles is the following:

First find a spanning tree. Then each arc not in the tree generates a cycle in the network. These cycles from a complete set, in the sense that all flows which satisfy the node flow conditions can be represented as superpositions of flows in these cycles.

Other choices of the complete set of cycles can be made and are often more efficient. There are two general criteria in this choice. Firstly, it is useful to separate out the paths or cycles of low resistance. Secondly, the efficiency is improved if the cycles have minimum overlap so that the Hessian matrix of the objective function has the least number of off-diagonal terms. These questions are discussed by Osiadazc.

Let $v_{l}$ be the flow in cycle $l$. Define the arc-cycle incidence matrix by

$$
b_{l k}=\left\{\begin{aligned}
1 & \text { if arc } k \text { is in cycle } l \text { in the same direction, } \\
-1 & \text { if arc } k \text { is in cycle } l \text { in the opposite direction, } \\
0 & \text { otherwise. }
\end{aligned}\right.
$$

Then $q_{k}=\sum_{l} v_{l} b_{l k}$ and

$$
\mathscr{H}=\sum_{k \in \mathscr{A}^{+}} \psi_{k}\left(\sum_{l} v_{l} b_{l k}\right)
$$

where $\mathscr{A}^{+}$is the set of arcs in the extended network and $\psi_{k}=-q_{k} P_{0}=-\lambda P_{0}$ for the dummy arc.

The problem then is an unconstrainted minimisation over the arc flows $v_{l}$. We can now show that the Hessian of $\mathscr{H}$ is positive definite so that there is a unique minimum value for $\mathscr{H}$. The quadratic form generated by 
the Hessian is

$$
\begin{aligned}
\sum_{i, j} v_{i} v_{j} \frac{\partial^{2} \mathscr{H}}{\partial v_{i} \partial v_{j}} & =\sum_{i, j} \sum_{k \in \mathscr{A}^{+}} v_{i} b_{i k} v_{j} b_{j k} \psi_{k}^{\prime \prime}\left(\sum_{l} v_{l} b_{l k}\right) \\
& =\sum_{k \in \mathscr{A}^{+}} q_{k}^{2} \psi_{k}^{\prime \prime}\left(q_{k}\right)>0,
\end{aligned}
$$

since $\psi_{k}^{\prime \prime}>0$

\section{A numerical example}

To illustrate the descriptions above, we include a simple numerical example. We consider a pipe network as shown in the diagram.

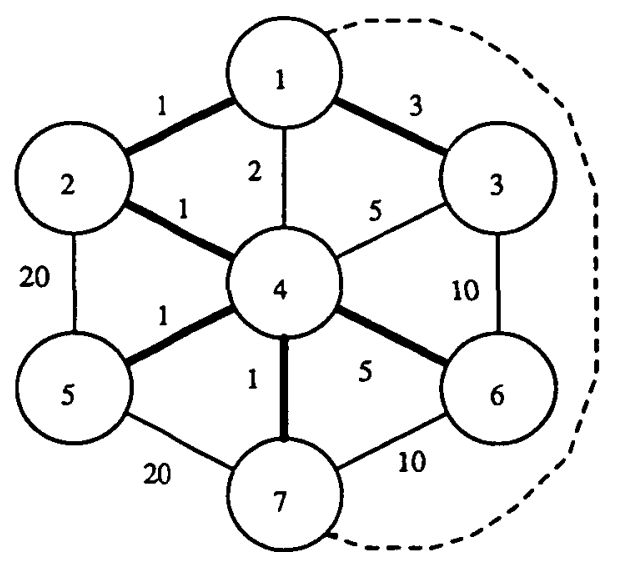

Suppose that the pressure at node number 1 is set at 1 , and the pressure at node 7 is set at 0 . The relation between the flow $q$ and the pressure difference $p$ is given by

$$
p_{k}=\alpha_{k} q_{k}^{2}
$$

This is a typical form used for flow at moderately high speeds. The values of $\alpha$ for the different arcs are shown on the diagram. The dotted arc between node 7 and node 1 is the dummy arc used for the loop formulation.

The problem was solved using GAMS (General Algebraic Modeling System) [1] as a front end to MINOS. The processing times were $0.016 \mathrm{~min}$ - 
utes for the nodal method and 0.018 minutes for the loop method on a 486 personal computer.

The nodal method used was exactly as described above, and no variations are available. For the loop method, the implementation depends on the choice of loops. The arcs shown in the diagram with heavier lines are the ones that define the spanning tree used to construct the loops. The separate addition of each arc not in the tree then defines a loop. Where the loop consists of only three arcs, it was accepted. Where the loop was larger, it was reduced in length if possible by "subtracting" from it a previous loop. Thus, for example, the loop through nodes $1-2-4-3-1$ is replaced by 1-4-3-1, achieved by subtracting the loop 1-2-4-1. A more complete description of a suitable algorithm for this construction is given by Osiadacz. The final set of loops chosen was:

$3-4-6-3$

4-7-6-4

$1-2-4-7-1$

If $m$ is the total number of arcs (including the dummy arc) and $n$ the number of nodes, the number of arcs in the spanning tree is $n-1$ and therefore the number of loops must be $m-n+1$, in this example $13-7+1=7$.

The results for the two methods were identical within the accuracy reported. The value for the objective was found to be 0.8035 . The pressures at nodes 1 to 7 were $1.000,0.769,0.752,0.617,0.610,0.602,0$, respectively.

\section{References}

[1] A. Brooks, D. Kendrick, and A. Meeraus, GAMS A User's Guide (The Scientific Press, San Francisco, 1988).

[2] R. Fletcher, Practical Methods of Optimization, Vol. 2 (John Wiley \& Sons, 1981).

[3] A. J. Osiadacz, Simulation and Analysis of Gas Networks (E. \& F. N. Spon Ltd., London, 1987).

[4] Y. J. Wang, "Solving mine vertilation networks with fixed and non-fixed branches", Mining Engineering (1990) 1342-1346. 